\title{
ROAD: domestic assistant and rehabilitation robot
}

\author{
Isela Carrera - Héctor A. Moreno - Roque Saltarén • \\ Carlos Pérez $\cdot$ Lisandro Puglisi $\cdot$ Cecilia Garcia
}

\begin{abstract}
This study introduces the concept design and analysis of a robotic system for the assistance and rehabilitation of disabled people. Based on the statistical data of the most common types of disabilities in Spain and other industrialized countries, the different tasks that the device must be able to perform have been determined. In this study, different robots for rehabilitation and assistance previously introduced have been reviewed. This survey is focused on those robots that assist with gait, balance and standing up. The structure of the ROAD robot presents various advantages over these robots, we discuss some of them. The performance of the proposed architecture is analyzed when it performs the sit to stand activity.
\end{abstract}

Keywords Robotic rehabilitation - Robotic assistant . Sit to stand activity

\section{Introduction}

One of the major problems that the society has to face toward the next decades is the quality of life and autonomy of people with disabilities.

Statistics show that the number of people with disabilities in Spain is increasing due to aging [13, 14]. For this reason, the development of assistive technology is an important task. Robots for rehabilitation and assistance can highly improve the quality of life of disabled people.
Robots for the disabled are generally classified into therapy robots and assistance robots. Therapy robots have two simultaneous users: the disabled person and the physical therapist. A robot is a good alternative for applying in physical therapy, because once the robot is properly adjusted, it can apply a consistent therapy for long periods of time without any effort on the part of the physiotherapists. Furthermore, robot sensors can measure the work performed by the patient and thereby determine when the patient could continue progressively with the therapy [12].

Assistance robots for manipulation can be fixed platforms (on a table, kitchen, bed, etc.) or portable (fixed to a wheelchair) to hold or move objects, or interact with other devices and equipments [9]. Assistance robots for mobility can be classified into two main parts: electric wheelchairs, equipped with navigation systems, and mobile robots, such as automated walkers, which support disabled persons in order to prevent mishaps and provide stability.

In this study, the concept design and analysis of a novel robotic system for rehabilitation and assistance for disabled persons are presented. Based on statistical information on the types of disabilities most common in Spain, the different tasks that the device should be able to execute have been determined. The robot presented here is intended to help in the rehabilitation process of a disabled person in tasks such as gait, balance, and standing up, and it is analyzed for the sit to stand (STS) activity.

\section{Methods}

\subsection{Disability and population}

A disability is defined as physical or mental impediment that substantially limits a person's movements, senses or 
Table 1 People with disabilities in industrialized countries

\begin{tabular}{lcr}
\hline Country & $\begin{array}{l}\text { \# People with } \\
\text { disability }(\%)\end{array}$ & \multicolumn{1}{l}{$\begin{array}{l}\text { \# Elderly people } \\
(\%)\end{array}$} \\
\hline France & $5,146,000(8.3)$ & $12,151,000(19.6)$ \\
USA & $52,591,000(20)$ & $35,000,000(12.4)$ \\
Great Britain & $4,453,000(7.3)$ & $12,200,000(29.5)$ \\
The Netherlands & $1,432,000(9.5)$ & $2,118,808(13.4)$ \\
Spain & $3,528,220(8.9)$ & $6,936,000(17.6)$ \\
Japan & $5,136,000(4.3)$ & $44,982,000(35.7)$ \\
Korea & $3,195,000(7.1)$ & $16,300,000(36.0)$ \\
\hline
\end{tabular}

activities. In the last decades, the number of disable people has significantly increased. In particular, in industrialized countries, it is mainly caused by the increased of live expectancy. Table 1 shows the number of people with disabilities and elderly people in some industrialized countries [7].

Low birth rate and advancement in health care are key factors that have contributed to the growth of the aging population, and it is expected that it will duplicate by the year 2030. This increasing segment of population will generate changes for the demand in health care, care giving, and medical expenses.

Therefore, technical assistive aids such as personal and service robots could be a key factor in solving this demand.

\subsection{Disability survey in spain}

Spain has a remarkable aging process in its population, and as a consequence, Spain has experienced an increase on the disable population, as reported in the EDAD 2008 (acronym in Spanish for Survey on Disabilities, Personal Autonomy and Dependency Situations) [14] and in EDDS 1999 (acronym in Spanish for the Survey on Disabilities, Deficiencies and Health Status) [13].

The report shows that there are 3.5 million of people with some type of disability or limitation in Spain, , who among $59.8 \%$ are women. The percentage of disabilities in terms of age is slightly higher up to 44 years of age in males. This figure is reversed after 45 years of age and this difference increases with age, see Fig. 1. Of all the disable population, the $67.2 \%$ presents limitations in moving or carrying objects.

From the EDDS 1999 [13], it was also found that:

- There are two main groups of displacement activities: (a) moving around the home and (b) activities of standing up, sitting down, and lying down.

- In the elderly population, it is observed that capabilities of the displacement and the usage of arms and hands are strongly affected with age.

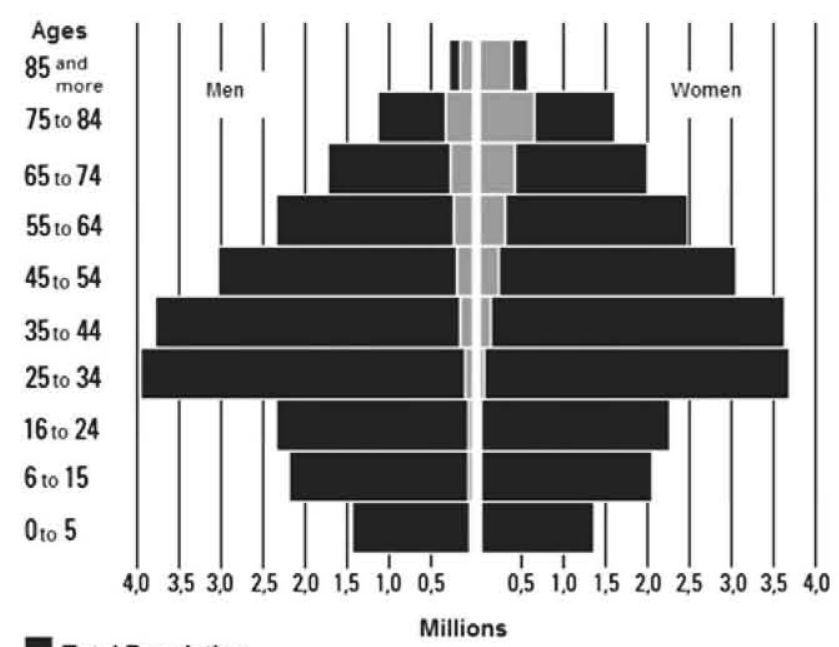

Total Population

Population with disabilities

Fig. 1 Pyramid of population

Table 2 Disability types in Spain (rates per thousands)

\begin{tabular}{lll}
\hline & Man & Woman \\
\hline Mobility & 42.6 & 77.5 \\
Domestic life & 29.5 & 69.2 \\
Self care & 31.3 & 55.3 \\
Hearing & 21.9 & 28.4 \\
Vision & 17.8 & 28.4 \\
Communication & 16.3 & 18.6 \\
Application of learning and knowledge & 12.7 & 17.1 \\
$\quad$ to develop tasks & & \\
Interaction and personal relationships & 14.0 & 15.4 \\
Total & 72.6 & 106.3 \\
\hline
\end{tabular}

- The activities of standing up, laying down, moving around inside the home, and carrying light objects are closely related.

- Most falls of elderly people occur inside the home.

$55.3 \%$ has problems related with domestic tasks and $48.8 \%$ with care and personal health tasks. The main cause of disability ( $42 \%)$ is the problems related with bones and articulations (see Table 2).

$88.9 \%$ of the population with disabilities receives either technical or personal care. Disabled people who do not receive any care, need rather technical $(27.3 \%)$ than personal assistance $(16.9 \%)$.

From the population with disabilities, 2.8 millions $(6.7 \%$ of the total in population Spain) have some type of disability that affects directly their Activities of Daily Living (ADL). $81.7 \%$ receives some kind of assistance that let them to reduce the severity of their disability. However, $19.3 \%$ does not receive any type of assistance. 
As it was mentioned before, more than 2 million of people has personal care and supervision. The profile of a typical caregiver is a woman between 45 and 65 years of age, and the $78.8 \%$ is $24 / 7$ live-in care giving. However, the caregiver may find some difficulties to accomplish their tasks, mainly caused by their physical limitation and fatigue.

Some conclusions can be drawn from the information on the surveys: the development of robotic systems for disabled persons is important because of the expansion of the handicapped community due to the increase in life expectancy; this means that elderly are the main consumers of these applications. The main issues that the robotic device is to solve are related to moving and transportation, helping with balance and gait, and standing up. However, we found it necessary to allow the patient to keep using his or her own strength in these activities. The robot can be a great aid for therapists and especially for caregivers, where the robot would carry the weight of the patient in order to make up for the lack of physical strength of the caregiver. The robot can be installed in a rehabilitation center or at home, and can help in the rehabilitation of ADLs.

\subsection{Assistance robots in rehabilitation}

Three schemes of operation have been considered for assistance robots: serial robots mounted on a workstation, serial robots mounted on a wheelchair, and autonomous mobile robots. In the case of serial robots mounted on a workstation, the history begins with the robot designed by Roesler in Heidelberg, East Germany in the mid 1970s. The system consisted of a manipulator with five degrees of freedom placed in a specially adapted desktop environment using rotating shelves units [30]. Other examples of this type of robots are the DeVAR robot (Desktop Vocational Assistive Robot) and the RT robot. DeVAR consisted of an industrial Puma 260 manipulator, mounted upside down on an overhead track [10]. The RT robot was a modified SCARA manipulator. The RT had two rotational joints with vertical axis that allowed the arm to move through the horizontal plane, and an additional degree of freedom in the vertical axis [17].

One of the most commercially successful robots was Handy 1 by Rehab Robotics from the United Kingdom. The project was initially designed to help people who have serious problems eating on their own. Recently, system extensions have been made to apply makeup, wash up, and shave [32]. Among the mobile robots, it is found that the Movar robot that is essentially the DEVAR robot with wheels. The mobile base has sophisticated omni-directional wheels [8].

Another robot of this type is the KARES II, which includes the use of an optical mouse, Haptic equipment, and a robotic arm. The arm has been assembled in a number of different configurations, but mainly on a remote mobile base [3]. Another notable project is the research from Bath Institute of Medical Engineering with the introduction of the Wessex robot, mounted on an automated mobile base [18].

The most notable robots mounted on wheelchairs are Manus and Raptor. Manus work began in 1984. Manus is a sophisticated robot manipulator that can be mounted on different wheelchairs. It has 7 degrees of freedom and a simple claw [11]. Moreover, Raptor is similar to Manus except for its price, a third of that of Manus [11, 25].

\subsection{Therapy robots}

The use of robots for facilitating the motion in rehabilitation therapy to stroke patients has been one of the fastest growing areas of research in recent years. The reason for this growth is the potential to provide effective therapy at a low, acceptable cost.

It is known that by exercising the affected part, it could recover some degree of functionality $[24,29]$. A Robot could be used for replicating the exercises provided by the therapist, but it also has the potential to reproduce other regimes that would not be easily carried out by a human being. Some of the robots with these abilities are the MIME System from VA Palo Alto that allows the movement of the affected and the unaffected limbs [31], and the the ARM and GENTLES [2] projects. On the other hand, a rehabilitation robotic system driven by pneumatic swivel modules was presented in [26, 27]. This robot is intended to assist in the treatment of stroke patients by applying the proprioceptive neuromuscular facilitation method.

Other examples of commercial robots for therapy are the InMotion Arm Robot, based on the pioneering MIT-Manus [23], and the ARMEO $^{\circledR}$ [15] series system.

Recently, some works have been focused on gait and balance rehabilitation for stroke patients. They are able to support patient's body, while he or she maintain a nearly natural walk and can concentrate on other activities. Within the group of gait rehabilitation, the walkaround system helps to walk to people who have suffered from hemiplegia or other diseases that require assistance in posture [34].

Other highly developed devices for rehabilitation and gait balance are WHERE I and WHERE II. WHERE I is a mobile robot that assists with gait, it contains one rotational degree of freedom arm manipulator that adjusts to different heights and sizes and supports the body. WHERE II is a mobile vehicle that consists of four pneumatic bars that are adjusted to each side of the body [21].

There are commercial robots for children called SAM and SAM-Y that help in gait rehabilitation. SAM is a technological transfer done by Enduro Medical 
Technology. SAM allows patients to stand and walk around without the help of a physical therapist [16]. The Lokomat ${ }^{(B)}$ is another example of commercially available assistant for gait rehabilitation. It is a driven gait orthosis that automates locomotion therapy on a treadmill [20].

One of the most complex works is KineAssist which is a robotic device for gait training and balance. KineAssists provides a weight partial support to the body in the torso. It provides various motion axis in the trunk and pelvis. The KineAssist leaves the legs free to allow a physical therapist work freely. The robot can follow the patient walking motion in different directions such as straight, turning or climbing a step. It can also catch the patient if he or she starts to fall down or assist in the therapy to stand up.

In addition to the development of gait rehabilitation devices, there are those that help to stand up from the sitting down position. Chugo et al. [4] propose a design focused on elderly people that need assistance for standing and walking in their daily lives. The system consists of a cushioned support with three degrees of freedom and a gait system. The support is actuated by a manipulation mechanism made up of a four bar system.

\subsection{Description of ROAD}

The purpose of ROAD robot is to assist the user in standing up, sitting down, and carrying out locomotion tasks. This robot is intended to interact with the user in the same environment (hospital or home). Its design guarantees the integrity and safety of the user and the elements around. The robot is suspended from the ceiling through tracks (see Fig. 2). This idea shows some advantages compared to other robots that move on the floor. These advantages are: less risk of collision with the disabled person, better performance as support for balance, better access to high places, no need of a navigation system for detecting objects on the floor, minimizes the use of space and adaptable to perform different tasks using different assembly tools at the end of the robot.
The robot is composed of three links connected by three joints. They are a traction system on the rails, a rotational joint with vertical axis, and the actuated prismatic joint that moves up and down the arm support.

The rail traction system is located on the ceiling and supports the entire structure. Different types of tools can be fixed on the arm support, depending on the task to be accomplished.

The rails are located on the roof according to a predefined route in the house. The rails can be curved and in some cases, a special mechanism can be used for making rail changes with turntables and exchangers. The carriages are based on Ceiling Hoists, a well known technology used for transporting handicapped persons.

By moving the two prismatic joints, and actuating dependently, it is possible to provide a holding bar, a harness, a clamp or a special designed support for carrying out a more specific task. Some components are available commercially and commonly used, such as rails on the ceiling, the prismatic actuators, etc., however, the integration of these components in the described manner offers the advantage that concerns its current use.

\subsection{Applications}

The main application of this system is the assistance in supporting the user for the different ADLs, for example assisting with gait, as shown in Fig. 3a).

In addition, some users with limitations of leg movement require staying in a static position, maintaining balance, and at the same time carry out other rehabilitation tasks. These users can lose stability and fall down. In order to avoid accidents, a therapist is required to stay by their side (Fig. 3b).

Another common rehabilitation application for patients is the training of motion patterns that are frequently used in the patients daily living, but that have been waning because of their illness. For that reason, the therapist guides the

Fig. 2 ROAD components
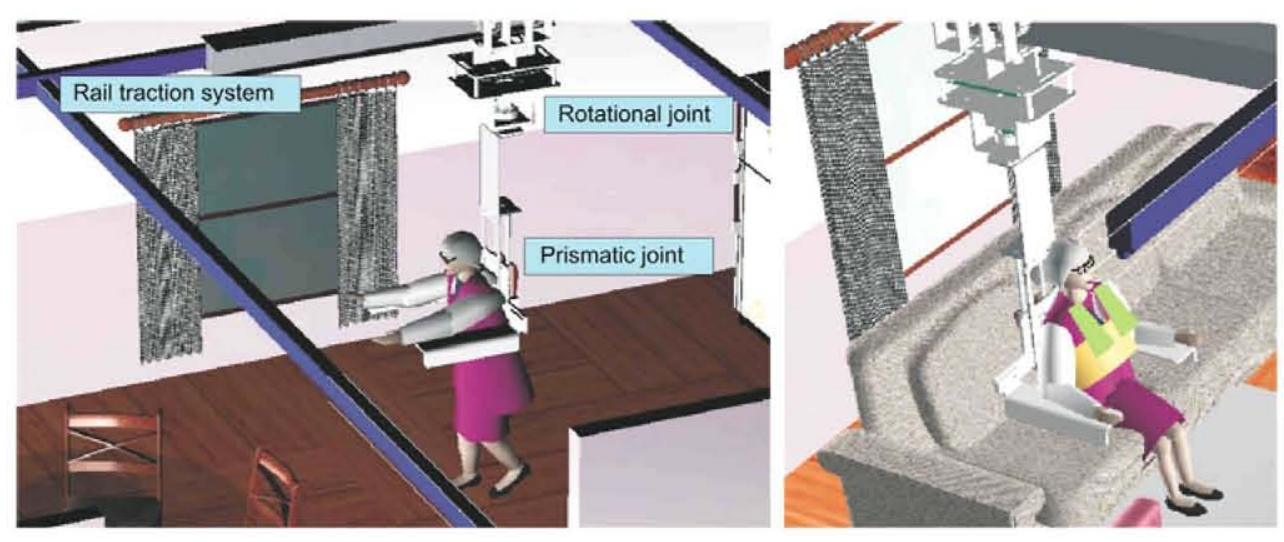

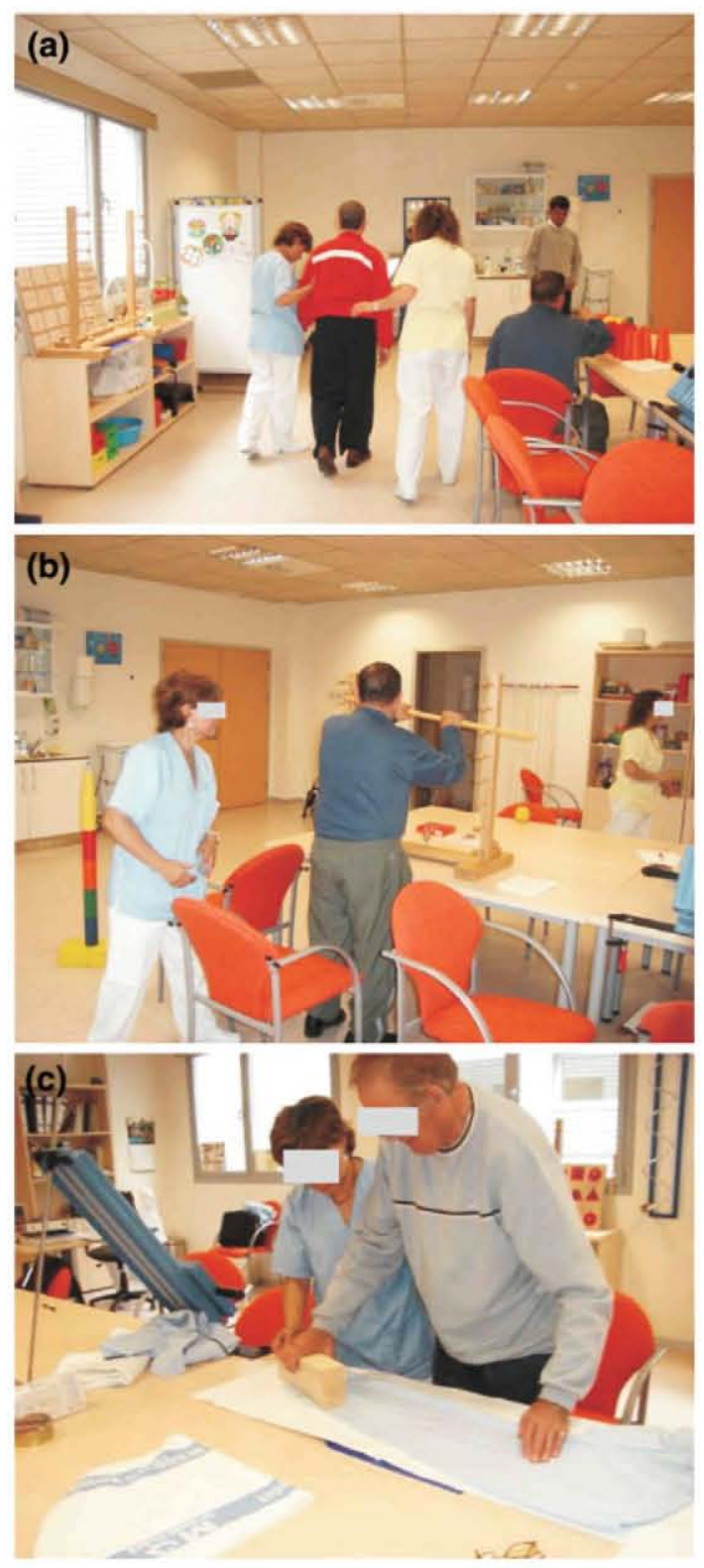

Fig. 3 Human assistance for disabled persons. a Therapist assisting with gait, b therapist assisting with balance in static standing, c therapist guides the patient in a movement pattern activity corresponding to ironing clothes

patient's limb in a way that it simulates the activity in need of relearning, such as ironing, as shown in Fig. 3c.

Depending on the robot's modularity, a device to support the user can be installed in the last element. The robot is programmed in such a way that the support follows a convenient trajectory, which produces predetermined motions in different joints, such as the desired exercise for rehabilitation. Because of the possibility of adapting different tools to the robot's end effector, it can assist the gait of the user through the use of a harness as a support tool for the weight of the body, so that the user's hands remain free during the gait or in a static position of balance while carrying out ADLs such as cooking or washing, without worrying about weight. Another end effector could be a clip or hook to help the user to load some heavy object. It can be stated that due to the robot's sensors, the system has the ability to obtain information, for example the strength or torque of the user. This information is highly important since it could be processed and considered in the following movements of the robot.

Considering the possibility that the robot follows complex trajectories, with positions, speed, and predefined forces of high precision and repeatability, other application of the proposed robot is to guide a user's limb for learning or training in the corresponding ADL tasks. An example of a complex trajectory is standing up since it is considered as one of the main problems to resolve. The path of the gait has been studied in the past. A robot with the appropriate end effector will be capable of reproducing the trajectory to help the patient's body to follow it and use the remaining strength without exhausting the patient's physical ability. The robot would even have the ability to provide assistance from the squatting to the standing position in case the user needs to pick something up from the floor.

\subsection{Modeling of ROAD}

In this section, the mathematical modeling of the robot is presented. The kinematic and dynamic analysis of ROAD is performed. The information obtained from this analysis allows determining the performance of the mechanism under different conditions in the design variables and the tasks to be accomplished.

\subsubsection{Kinematic analysis}

Considering the kinematic scheme of ROAD shown in Fig. 4, the kinematic analysis of ROAD robot is done in this section.

The fixed reference frame is on the ceiling. For our purposes, the $x-y$ plane is parallel to the sagittal plane. The joint variable $q_{1}$ corresponds to the prismatic joint that provides horizontal movement to the system. The joint variable $q_{2}$ corresponds to the rotational joint whose axis is parallel to the $y$ axis of the reference frame. Finally, the joint variable $q_{3}$ corresponds to that joint that provides vertical movement.

Direct position analysis The direct position analysis consists of determining the position of the handle, $\mathbf{r}_{t}=$ $\left[\begin{array}{lll}r_{t x} & r_{t y} & r_{t z}\end{array}\right]^{\mathrm{T}}$, given the values of the joint variables vector, $\mathbf{q}=\left[\begin{array}{lll}q_{1} & q_{2} & q_{3}\end{array}\right]^{\mathrm{T}}$ From Fig. 4 , the vector $\mathbf{r}_{t}$ can be obtained in the following form: 

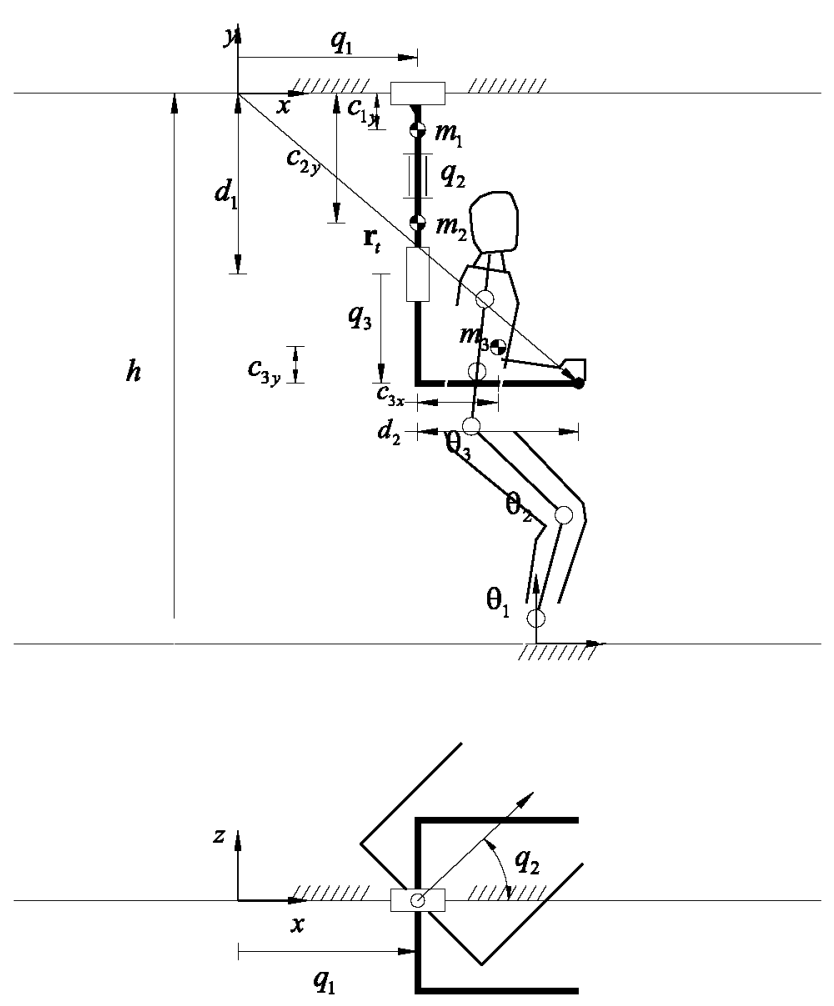

Fig. 4 Kinematic scheme of the ROAD robot

$\mathbf{r}_{t}=\left[\begin{array}{c}q_{1}+d_{2} \cos \left(q_{2}\right) \\ -\left(q_{3}+d_{1}\right) \\ d_{2} \sin \left(q_{2}\right)\end{array}\right]$

Parameters $d_{1}$ and $d_{2}$ depends on the geometrical characteristics of the system.

Direct velocity analysis The direct velocity analysis consists of determining the velocity of the handle, given the actuators velocities. Deriving (1), we can obtain the velocity model of the system in the following form:

$\dot{\mathbf{r}}_{t}=\mathbf{J} \dot{\mathbf{q}}$

where $\dot{\mathbf{r}}_{t}$ and $\dot{\mathbf{q}}$ denote the time derivatives of $\mathbf{r}_{t}$ and q, respectively. The Jacobian matrix is:

$\mathbf{J}=\left[\begin{array}{ccc}1 & -d_{2} \sin \left(q_{2}\right) & 0 \\ 0 & 0 & -1 \\ 0 & d_{2} \cos \left(q_{2}\right) & 0\end{array}\right]$

Direct acceleration analysis Deriving (2), the acceleration of the handle, $\ddot{\mathbf{r}}_{t}$, can be computed given the velocities and accelerations in the joints, $\dot{\mathbf{q}}$ and $\ddot{\mathbf{q}}$. The direct acceleration analysis is obtained by the following equation:

$\ddot{\mathbf{r}}_{t}=\mathbf{J} \ddot{\mathbf{q}}+\dot{\mathbf{J}} \dot{\mathbf{q}}$

where matrix $\mathbf{J}$ is the time derivative of (3) and has the following form:
$\dot{\mathbf{J}}=\left[\begin{array}{ccc}0 & -d_{2} \cos \left(q_{2}\right) \dot{q}_{2} & 0 \\ 0 & 0 & 0 \\ 0 & -d_{2} \sin \left(q_{2}\right) \dot{q}_{2} & 0\end{array}\right]$

Inverse position analysis The inverse position analysis consist of determining the joint variables, $q_{1}, q_{2}$ and $q_{3}$, given the position of the handle $\mathbf{r}_{r}$. The solution is obtained directly:

$q_{1}=r_{t x}-d_{2} \sqrt{1-\left(\frac{r_{t z}}{d_{2}}\right)^{2}}$

$q_{2}=\arcsin \left(\frac{r_{t z}}{d_{2}}\right)$

and

$q_{3}=-d_{1}-r_{t y}$

Inverse velocity analysis In order to obtain the required joint velocities for a desired velocity in the handle, Eq. 2 is solved for $\dot{\mathbf{q}}$ :

$\dot{\mathbf{q}}=\mathbf{J}^{-1} \dot{\mathbf{r}}_{t}$

Inverse acceleration analysis Inverse acceleration analysis is obtained in the following form:

$\ddot{\mathbf{q}}=\mathbf{J}^{-1}\left(\ddot{\mathbf{r}}_{t}-\dot{\mathbf{J}} \dot{\mathbf{q}}\right)$

\subsubsection{Dynamic analysis}

In this section, the dynamic analysis of the ROAD robot is performed. Lagrange method is applied for the analysis because it allows formulating the equations of motion by using a set of generalized coordinates (i.e., the joint variables).

The Lagrange equations of motion are given in the following way [33]:

$\frac{\mathrm{d}}{\mathrm{d} t}\left[\frac{\partial L}{\partial \dot{q}_{i}}\right]-\frac{\partial L}{\partial q_{i}}=\tau_{i}$

for $i=1,2,3$, where $\tau_{i}$ is the actuator torque at joint $i$ and $L$ is the Lagrangian function.

The Lagrangian function is defined as the difference between kinetic and potential energy:

$L=K-U$

From the scheme in Fig. 4, the following expressions of energy for each link can be found:

$K_{1}=\frac{1}{2} m_{1} \dot{q}_{1}^{2}$

$K_{2}=\frac{1}{2} m_{2} \dot{q}_{1}^{2}+\frac{1}{2} I_{2} \dot{q}_{2}^{2}$ 


$$
\begin{aligned}
K_{3}= & \frac{1}{2} m_{3} \dot{q}_{1}^{2}-m_{3} \dot{q}_{1} \dot{q}_{2} c_{3 x} \sin \left(q_{2}\right)+\frac{1}{2} m_{3} \dot{q}_{2}^{2} c_{3 x}^{2} \\
& +\frac{1}{2} I_{3} \dot{q}_{2}^{2}+\frac{1}{2} m_{3} \dot{q}_{3}^{2} \\
U_{1}= & -m_{1} c_{1 y} g \\
U_{2}= & -m_{2} c_{2 y} g \\
U_{3}= & -m_{1}\left(d_{1}+q_{3}-c_{3 y}\right) g
\end{aligned}
$$

where $m_{1}, m_{2}$, and $m_{3}$ are the masses of the three links, respectively; $I_{2}$ and $I_{3}$ are the inertia moments of links 2 and 3 in the $y$ axis; $c_{1 y}, c_{2 y}, c_{3 y}$, and $c_{3 x}$ are the coordinates of the center of mass of each link; and $g$ is the gravity.

With the previous expressions, the Lagrangian equation of the system is obtained:

$$
\begin{aligned}
L & =\frac{1}{2} m_{2} \dot{q}_{1}^{2}+\frac{1}{2} m_{2} \dot{q}_{1}^{2}+\frac{1}{2} I_{2} \dot{q}_{2}^{2}+\frac{1}{2} m_{3} \dot{q}_{1}^{2} \\
& -m_{3} \dot{q}_{1} \dot{q}_{2} c_{3 x} \sin \left(q_{2}\right)+\frac{1}{2} m_{3} \dot{q}_{2}^{2} c_{3 x}^{2}+\frac{1}{2} I_{3} \dot{q}_{2}^{2}+\frac{1}{2} m_{3} \dot{q}_{3}^{2} \\
& +m_{1} c_{1 y} g+m_{2} c_{2 y} g+m_{3}\left(d_{1}+q_{3}-c_{3 y}\right) g
\end{aligned}
$$

From Eq. 11, the equations of motion of the ROAD are obtained:

$$
\begin{aligned}
\tau_{1} & =\left(m_{1}+m_{2}+m_{3}\right) \ddot{q}_{1}-m_{3} \ddot{q}_{2} c_{3 x} \sin \left(q_{2}\right) \\
& -m_{3} \dot{q}_{2}^{2} c_{3 x} \cos \left(q_{2}\right) \\
\tau_{2} & =\left(I_{2}+m_{3} c_{3 x}^{2}+I_{3}\right) \ddot{q}_{2}-m_{3} \ddot{q}_{1} c_{3 x} \sin \left(q_{2}\right) \\
\tau_{3} & =m_{3} \ddot{q}_{3}-m_{3} g
\end{aligned}
$$

\section{Results}

\subsection{Sit to stand biomechanics}

Rising from the chair is an important basic task of daily living. It requires coordination and accurate balance control. Moreover, it is a prerequisite for gait. Without the ability of rising from a chair, many potentially ambulant patient and elderly people will remain prisoners in their chair. Therefore, the capability of moving from a sitting position to a standing position (STS) has been regarded as an important indicator of an elderly person to be functionally independent. If standing is impaired, it increases the risk factor for falls [19].

The pattern and definition of the STS activity have been analyzed by many authors $[5,19,22,28]$ based on the observation and measurements of body markers. Aissaoui and Dansereau [1] present a detailed description of the STS cycle, based on the detection of events that separates the cycle into phases (see Fig. 5):
Forward momentum It represents $27 \%$ of the STS cycle, and corresponds to the transfer of the weight from the seat to the feet area.

Seat-unloading (7\% STS cycle). It is detected by fast positive changing in vertical ground reaction forces, and vertical acceleration of the body mass.

Ascending (39\% STS cycle). It begins at the seat off, where the ground reaction forces reaches its maximum. It starts with acceleration between 34 and $45 \%$ of STS cycle and a long period of deceleration (45-73\%) of STS cycle. The vertical upward movement is ended when the knee is fully extended.

Stabilization (27\% STS cycle) It is detected when the vertical ground reaction force fluctuation does not exceed $\pm 1 \%$ of the body weight.

Considering the results obtained in [28], the muscular activity of the lower extremity is computed using the SIMTK package [6]. The angular positions of the joints from [28], represents the relative angle between body segments, are expressed according to the settings of SIMTK.

The simulations obtains the forces generated by tibialis anterior, peroneus longus, soleus (SOL), gastrocnemius medialis (GMed), biceps femoris (BF), vastus medialis (VM), vastus intermedious (VI), vastus lateralis (VL), gluteus maximus (GMax), and erector spinae and their results are presented in Fig. 5. For this simulation, a healthy individual who is $1.8 \mathrm{~m}$ tall and $85 \mathrm{~kg}$ in weight was considered. The initial contact between the chair and the human is not considered.

As observed at the beginning of the STS cycle, the muscles with higher activity acts on the anterior leg; the VI, and VL muscles while on the other hand, the BF muscle relaxes. From the $45 \%$ of the cycle, the BF muscle begins its contraction and assists the vertical movement during the ascending segment of the STS cycle. From the 60\%, VI and VL begin their relaxation. It is also important to recall the activity of the SOL muscle during the STS cycle, which is mainly responsible for support and balance during the cycle.

Figure 5 a shows the values of joint angles, these angles are measured with respect to the initial position, and are denoted as $\theta_{1}^{*}, \theta_{2}^{*}$, and $\theta_{3}^{*}$. Figure $5 \mathrm{~b}$ shows the velocity of the joint variables. As can be seen, the trajectory is continuos in velocity, and the beginning and end are cero. The movement pattern was extracted from [28]. Figure $5 \mathrm{c}$ and d shows the motion and velocity of the robot's joints, respectively.

\subsection{Analysis of the performance of the robot} during the STS task

In order to determine the ability of the robot in assisting the elderly, an analysis of the robot's dynamics using the ADAMS multibody simulator was conducted. 

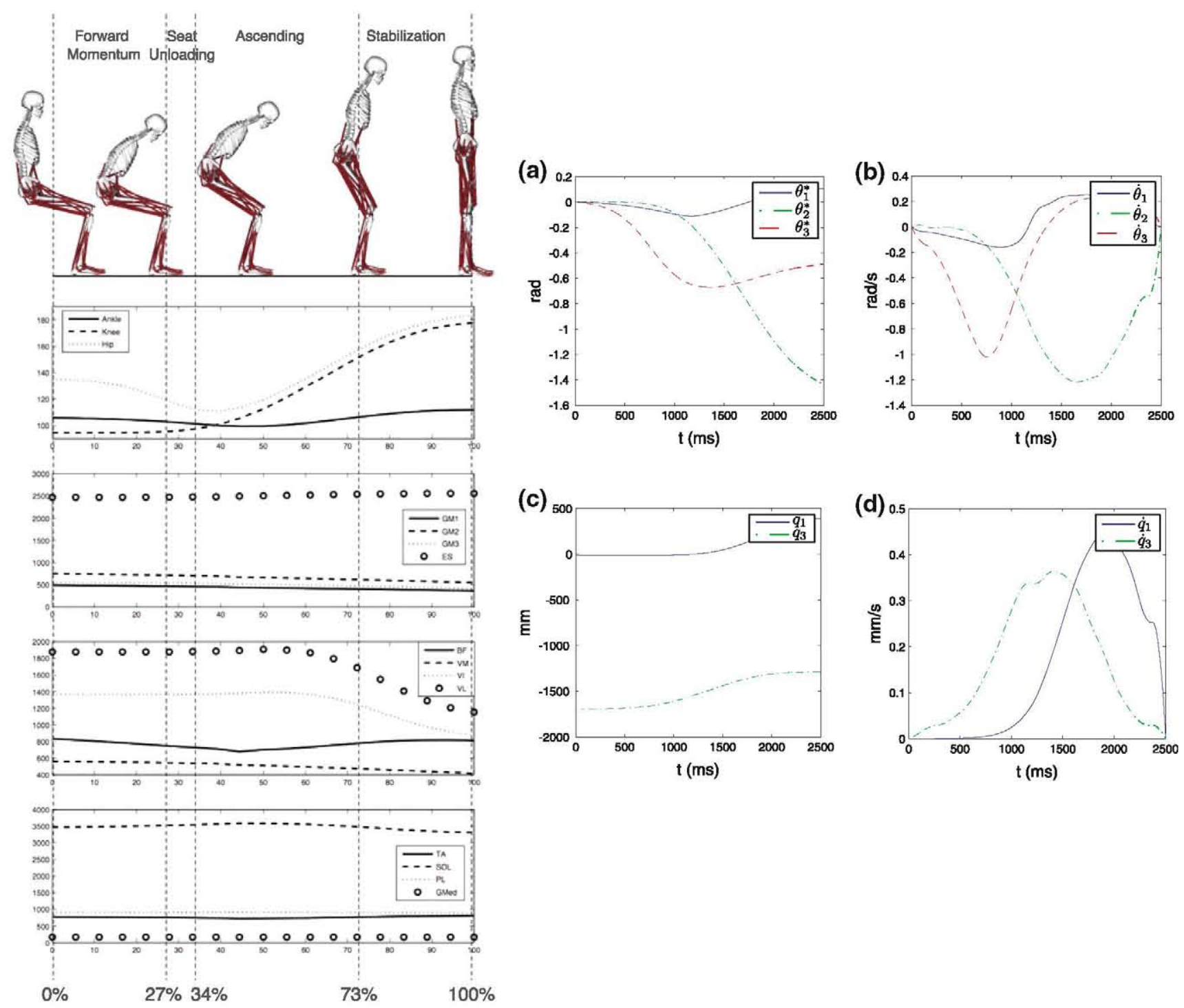

Fig. 5 Biomechanics and kinematics of the STS activity. Angles are in degrees and Forces in Newton in the biomechanics study. a and $\mathbf{b}$ present the positions and velocities of the leg joints. $\mathbf{c}$ and $\mathbf{d}$ show the positions and velocities of the robot's joints

The task consisted of lifting a dummy. When performing the STS task, we considered that the user must wear a harness in order to be lifted by the robot. The arm support is only intended to provide stability when the robot is assisting with gait.

Using the information obtained from the movement pattern that a person does during this operation, the movements required in the actuators to perform the task was determined by inverse kinematics.

The task was performed in $2.5 \mathrm{~s}$. Figure 6 is a sequence of images of the simulation in ADAMS. In this simulation, a dummy that $1.8 \mathrm{~m}$ tall and $85 \mathrm{~kg}$ in weight was considered.

On the other hand, Fig. $6 \mathrm{a}-\mathrm{c}$ shows the forces in $x-y$ in each of the leg joints. The sign depends on whether it is an action or reaction force. The major force generated is at the hip of about $-95 \mathrm{~N}$. The maximum force at the ankle is about $85 \mathrm{~N}$. This force is substantially lower if the person is not assisted by the robot, since a the person standing supports around $800 \mathrm{~N}$ at the ankle.

Figure $6 \mathrm{~d}$ and e shows the forces in the rail traction system. As shown in the figure, the maximum force in the beam is $1400 \mathrm{~N}$, on the other hand, the translational actuator must generate a force of almost $600 \mathrm{~N}$. On the other hand, we present the horizontal and vertical components of force in the joint $q_{3}$. The maximum force required in the actuator is $845 \mathrm{~N}$. This information allows determining the characteristics of the actuator/gear system.

\subsection{Robotic mockup}

In order to evaluate the concept design of the robot, a scaled model of the robotic system and its environment 


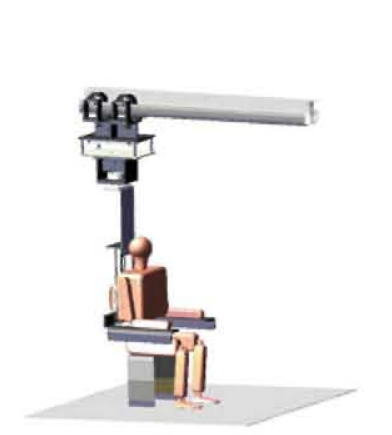

$I$

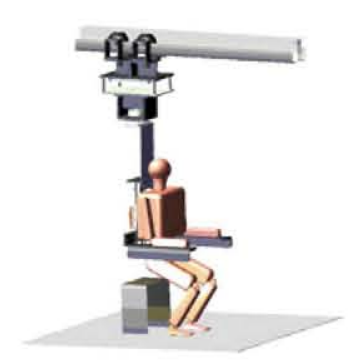

III

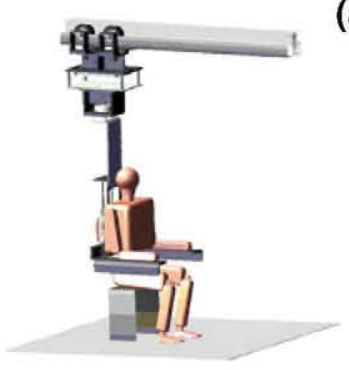

II

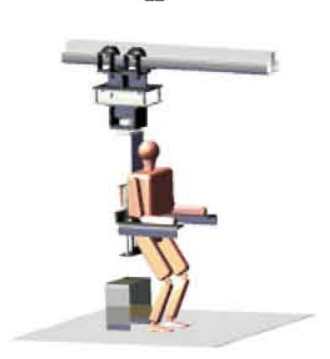

IV
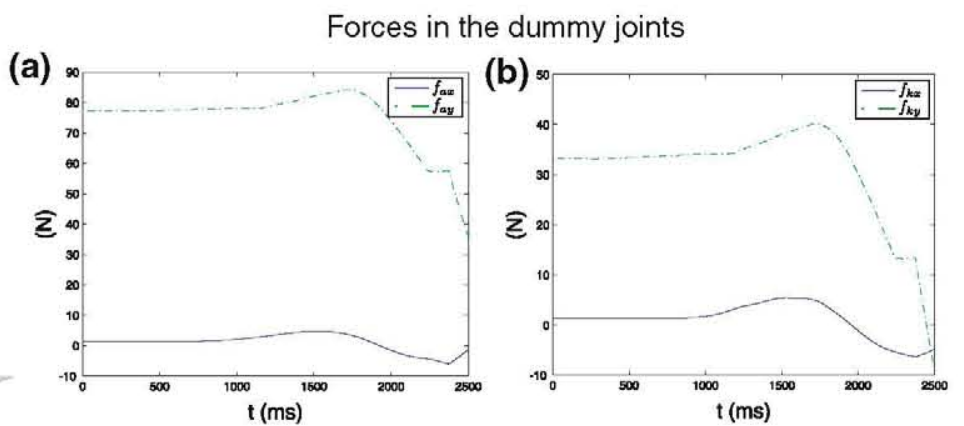

(c)

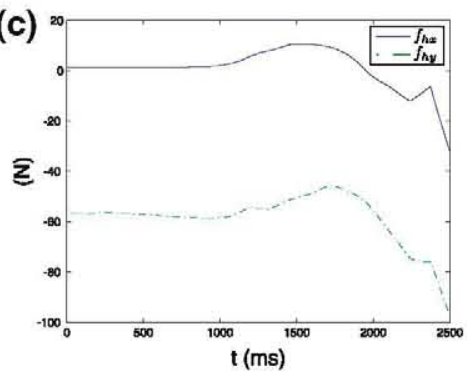

Forces in the robot joints
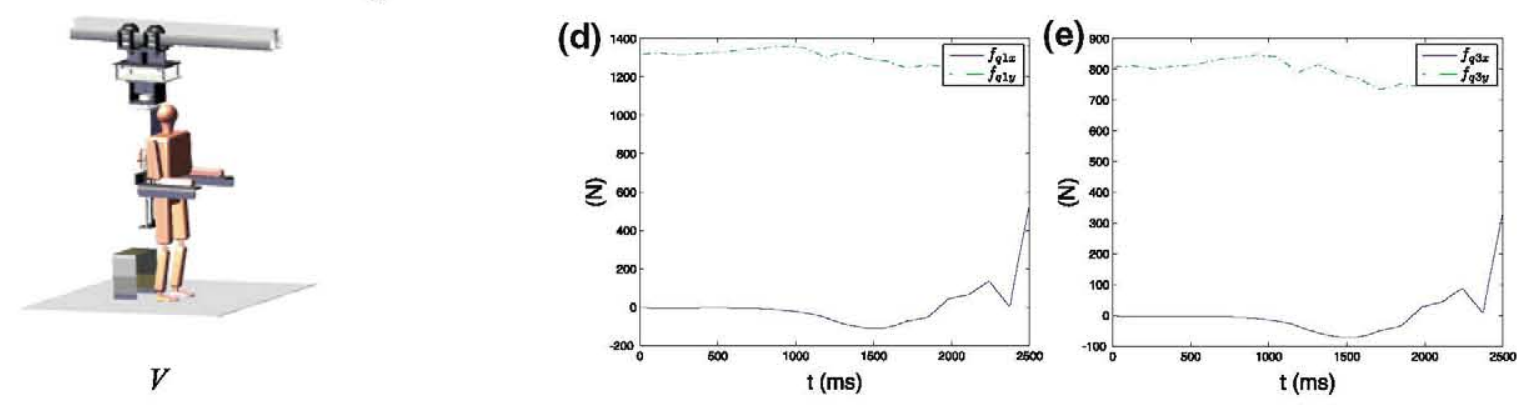

Fig. 6 ADAMS simulation. a-c Forces at the leg joints in the sagittal plane. $\mathbf{d}$ and $\mathbf{e}$ Forces at the robot's joints

have been constructed. This robotic mockup allows evaluating some aspects of the practical implementation of ROAD, such as dimension of the robot, possible collisions with its environment, route of the rails, etc.

It consists of two rooms (bathroom and kitchen) and a scale model of ROAD as can be observed in Fig. 7. The dimensions of both rooms have been obtained from a real house, named as Intelligent House, which is installed in the Telecommunication School of Technical University of Madrid. The Intelligent House is a project to extend the independent life of elderly people using Ambient Assisted Living services and Domotics technologies.

The scaled model of ROAD is completely actuated, i.e., all its joints have an electric motor. A Galil motion controller was used for synchronizing and controlling the movements of the motors. The software for controlling the system was developed in LabView. In addition, a small humanoid for demonstrating the motion synchronization when the robot is assisting with gait is included.
This robotic mockup also serves as a test bed of the software tools and algorithms for the autonomous operation of the robot in diverse scenarios.

\section{Discussion}

From the results of the surveys, it can be seen that the technical solution for assisting the impaired will be an important topic to cover in the coming years due to the increase in disable people. Some of the principal problems to be solved are related to displacement and transportation, balance, gait, and STS tasks. However, STS activity is considered to be the most critical among the rest.

The robot assistant could be of great use for therapist and caregivers, especially for carrying and treating patients.

Based on this aspect, a novel design that is able to assist people with limited motor functions is proposed in this study. This novel design called ROAD is focused on the assistance of STS activity, balancing and gait. It could be 


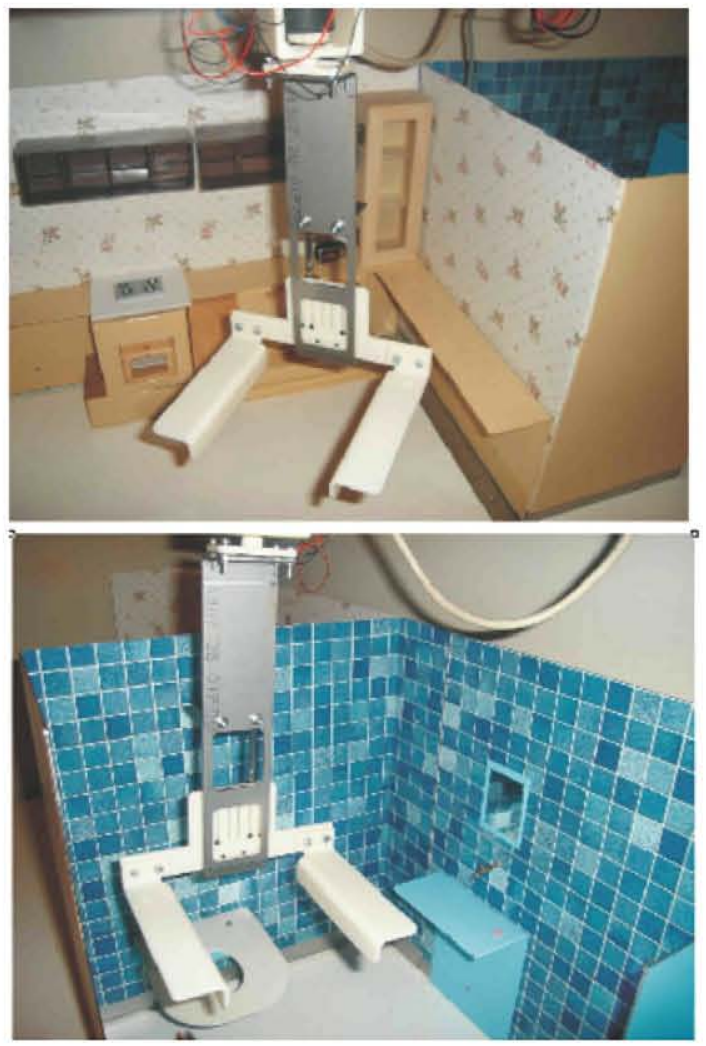

Fig. 7 Robotic mockup

installed in a rehabilitation center or at home in order to assist disabled people in their ADLs and rehabilitation therapy.

This proposal presents several advantages in the achievement of the desired objectives from robots that only move on the floor. These advantages are: there is less risk of collision with the person, better performance when it serves as a support for balance and better access to high places. Moreover, it is not necessary a navigation system for detecting objects on the floor. It also minimizes using free space in the room and is able to adapt in order to perform different tasks using different assembly tools at the end of the robot.

Acknowledgments The authors would like to thank the Spanish Government CICYT Project Ref. DPI2009-08778 for the financial support and the Comunidad de Madrid for supporting the project ROBOCITY2030-II Ref. P2009/DPI-1559. Ms. Carrera would also like to thank CONACYT Mexico for their financial support. The authors would like to thank Dr. Javier Lopez and the Hospital Infanta Sofia for their collaboration in this study.

\section{References}

1. Aissaoui R, Dansereau J (1999) Biomechanical analysis and modelling of sit to stand task: a literature review. IEEE Int Conf Syst Man Cyber 1:141-146. doi:10.1109/ICSMC.1999.814072
2. Amirabdollahian F, Loureiro R, Driessen B, Harwin W (2001) Error correction movement for machine assisted stroke rehabilitation. Integr Assist Technol Inf Age 9:60-65

3. Bien Z, Kim DJ, Stefanov DH, Han JS, Park HS, Chang PH (2002) Development of a novel type rehabilitation robotic system KARES II. Universal access assistive technology, Trinity Hall, University of Cambridge, Cambridge, pp 201-212

4. Chugo D, Mastuoka W, Jia S, Takase K, Asama H (2007) Rehabilitation walker with standing assistance. In: Rehabilitation robotics, 2007. ICORR 2007. IEEE 10th international conference, pp 132-137. doi:10.1109/ICORR.2007.4428418

5. Dehail P, Bestaven E, Muller F, Mallet A, Robert B, BourdelMarchasson I, Petit J (2007) Kinematic and electromyographic analysis of rising from a chair during a "sit-to-walk" task in elderly subjects: role of strength. Clin Biomech 22(10): 1096-1103

6. Delp SL, Anderson FC, Arnold AS, Loan P, Habib A, John CT, Guendelman E, Thelen DG (2007) OpenSim: open-source software to create and analyze dynamic simulations of movement. IEEE Trans Biomed Eng 54(11):1940-50. doi:10.1109/TBME. 2007.901024

7. der Loos HFMV, Mahoney R, Ammi C (2004) Great expectations for rehabilitation mechatronics in the coming decade. Adv Rehabil Robot 306:427-433

8. der Loos MV, Michalowski S, Leifer L (1986) Design of omnidirectional mobile robot as a manipulation aid for severely disabled. In: Foulds $\mathrm{R}$ (ed) Interactive robotics aid. World Rehabilitation Fund, New York, pp 61-63

9. der Loos MV, Reinkensmeyer DS (2008) Chapter 54: Health Care and Rehabilitation Robotics. In: Siciliano B, Khatib O (eds) Handbook of robotics, Springer-Verlag, Berlin, pp 1223-1251

10. der Loos HFMV, Wagner JJ, Smaby N, Chang K, Madrigal O, Leifer LJ, Khatib O (1999) Provar assistive robot system architecture. In: Proceedings of the ICRA'99: IEEE international conference on robotics automation, Vol 1-4, pp 741-746

11. Driessen BJF, Evers HG, van Woerden JA (2001) Manus-a wheelchair-mounted rehabilitation robo. Proc Inst Mech Eng $\mathrm{H}$ 215(H3):285-290

12. Emken J, Reinkensmeyer D (2005) Robot-enhanced motor learning: accelerating internal model formation during locomotion by transient dynamic amplification. IEEE Trans Neural Syst Rehabil Eng 13(1):33-39. doi:10.1109/TNSRE.2004.843173

13. Encuesta sobre discapacidades, deficiencias y estado de salud, 1999. Instituto Nacional de Estadística. España (1999)

14. Encuesta de Discapacidad, autonomía personal y situaciones de dependencia, EDAD 2008. Instituto Nacional de Estadística. España (2008)

15. Gijbels D, Lamers I, Kerkhofs L, Alders G, Knippenberg E, Feys $P$ (2011) The armeo spring as training tool to improve upper limb functionality in multiple sclerosis: a pilot study. J Neuroeng Rehabil 8:5

16. Goddar Space Flight Center, N.: Goddards cable-compliant joint technology gets patients up and walking with SAM (2008) http://ipp.gsfc.nasa.gov/SS-SAM.html. Accessed 22 Apr 2009

17. Hillman M (2004) Rehabilitation robotics from past to present-a historical perspective. Adv Rehabil Robot 306:25-44

18. Hillman M, Hagan K, Hagan S, Jepson J, Orpwood R (2002) Weston wheelchair mounted assistive robot-the design story. Robotica 20:125-132. doi:10.1017/S0263574701003897

19. Inkster L, Eng J (2004) Postural control during a sit-to-stand task in individuals with mild parkinson's disease. Exp Brain Res 154:33-38. doi:10.1007/s00221-003-1629-8

20. Jezernik S, Pfister A, Frueh H, Colombo G, Morari M (1999) Robotic orthosis lokomat: its use in the rehabilitation of locomotion and in the development of the biology-based neural controller. In : Annual IFESS conference, Ljubljana, pp 301-303 
21. KapHo S, JuJang L (2009) The development of two mobile gait rehabilitation. IEEE Trans Neural Syst Rehabil Eng 17(2): 156-166

22. Kouta M, Shinkoda K, Kanemura N (2006) Sit-to-walk versus sitto-stand or gait initiation: biomechanical analysis of young men. J Phys Ther Sci 18(2):201-206

23. Krebs H, Hogan N, Aisen M, Volpe B (1998) Robot-aided neurorehabilitation. IEEE Trans Rehabil Eng 6(1):75-87. doi: $10.1109 / 86.662623$

24. Krebs HI, Volpe BT, Aisen ML, Hening W, Adamovich S, Poizner H, Subrahmanyan K, Hogan N (2003) Robotic applications in neuromotor rehabilitation. Robotica 21:3-11

25. Mahoney RM (2001) The raptor wheelchair robot system. Integr Assist Technol Inf Age 9:135-141

26. Menchon M, Morales R, Badesa FJ, Domnech LM, Garca Aracl. N, Sabater JM, Prez C, Fernndez E (2010) Pneumatic rehabilitation robot: modelling and control. In: Proceedings for the joint conference of ISR 2010 and ROBOTIK 2010, Munich

27. Morales R, Badesa FJ, Domenech LM, Garca-Aracil N, Sabater JM, Menchon M, Fernandez E (2010) Design and control of a rehabilitation robot driven by pneumatic swivel modules. In: 3rd IEEE RAS and EMBS internacional conference on biomedical robotics and biomechatronics, IEEE catalog number: CFP10 BRB-DVD, Tokyo

28. Nuzik S, Lamb R, VanSant A, Hirt S (1986) Sit-to-stand movement pattern-a kinematic study. Phys Ther 66(11):1708-1713

29. Riener R, Nef T, Colombo G (2005) Robot-aided neurorehabilitation of the upper extremities. Med Biol Eng Comput 43:2-10. doi: $10.1007 / \mathrm{BF} 02345116$

30. Roeschel O (1996) Linked darboux motions. Math Pannon 7(2):291-301

31. Shor PC, Lum PS, Burgar CG, der Loos HFMV, Majmundar M, Yap R (2001) The effect of robotic-aided therapy on upper extremity joint passive range of motion and pain. Integr Assist Technol Inf Age Age 9:79-83

32. Topping M (2001) Handy 1 , a robotic aid to independence for severely disabled people. Integr Assist Technol Inf Age 9:142147

33. Tsai LW (1999) Robot analysis: the mechanics of serial and parallel manipulators. Wiley, New York

34. Veg A, Popovic DB (2008) Walkaround: mobile balance support for therapy of walking. IEEE Trans Neural Syst Rehabil Eng 16(3):264-269. doi:10.1109/TNSRE.2008.918424 